\title{
Der Grad der Polyploidie bei den Angiospermen in verschiedenen GroBarealen
}

\author{
G. Tischler \\ Botanisches Institut der Universität, Kiel
}

Eingegangen am 30. August, 1954

Im Jahre 1932 veröffentlichte HAGERUP eine sehr interessante Arbeit, in der er nachzuweisen suchte, daß die Anzahl der Polyploiden in einem Lande mit extremen Klimaverhältnissen stark vergrößert gegenüber derjenigen wäre, deren Pflanzen unter weniger extremen Verhältnissen wachsen. Er verglich Stichproben aus der Flora Timbuktus in Centralafrika einerseits mit dem Verhalten der Ericales im hohen Norden andererseits und fand in beiden Fällen eine ihm ungewöhnlich groß erscheinende Menge polyploider Arten. Darauf sprach er die Vermutung aus, daß dieser Grad von Polyploidie und die extremen Klimabedingungen korrelativ miteinander verknüpft wären. Diese Arbeit hat sich als außerordentlich fruchtbar erwiesen, und noch heute stehen wir in ihrem Banne. HAGERUPs Gedanke darf als bewiesen angesehen werden, auch wenn es wohl erlaubt ist, darauf hinzuweisen, daß wir das damals vorliegende Beweismaterial kaum mehr als zwingend anerkennen werden. Denn der Prozentsatz der Polyploiden, den man nach HAGerups Angaben für Timbuktu errechnet $(34,5 \%)$, ist durchaus nicht besonders hoch, und selbst, wenn wir zwei von ihm noch als diploid betrachtete Species (Farsetia ramosissima und Polygala triflora) als polyploid rechnen würden, kämen wir nur zu $41,4 \%$, einem Prozentsatz, der weit unter dem der doch gewiß nicht extremen Verhältnisse Mitteleuropas liegt. Was die Ericales anlangt, für die er bei 4 Gattungen (Kalmia, Empetrum, Arctostaphylos und Clethra, HaGeruP 1928) die nordischen Arten mit einer Chromosomenzahl aufdeckte, die das Doppelte der weiter südlich wachsenden Species war, so können wir gerade diese Gruppe kaum mehr als Beispiel für seine Lehre werten, da ein besonders großer Reichtum an Diploiden im Norden vorhanden ist. Er hat selbst 1928 wie auch später gezeigt, wie sehr sich diese oft den klimatischen Verhältnissen des Nordens angepaßt haben. Aber die Tatsache, daß die anfänglichen Stützen der Theorie jetzt durch bessere zu ersetzen sind, kann nichts gegen die Genialität der ursprünglichen Conception besagen.

Kurze Zeit darauf (1934) griff ich selbst in diese Fragen ein. Ich sagte mir, daß nur bei Zusammenfassung aller Arten eines Landes die unvermeidlichen Verschiedenheiten, die sich für die einzelnen Familien ergeben werden, ausgeglichen sein können. Und so würde uns vielleicht erlaubt sein, von der Gesamthöhe eines Polyploidiegrades in einem größeren Areal zu sprechen. Daraufhin habe ich nicht nur die Flora von Schleswig-Holstein ausführlich in bezug auf den Mindestsatz von Polyploiden behandelt, sondern mit dieser auch 
die Floren eines mediterranen Landes, nämlich von Sizilien, und gewisser nordischer Inseln (Faröer und Island) verglichen. Es ergab sich dabei ein starker Unterschied, der sich am besten im Sinne von HAGERUPs Lehre erklären ließ.

Seitdem ist eine Reihe ähnlicher Berechnungen durchgeführt, und weitere werden folgen. Dazu pflegen manche Autoren, in erster Linie Nicht-Cytologen, zu bemerken, daß das Beweismaterial deshalb nicht stichhaltig sein könne, weil die Bestimmung der Chromosomenzahl nur zum kleineren Teil in dem zu untersuchenden Lande vorgenommen wurde, und die Gefahr, daß dabei chromosomal verschiedene Rassen nicht berücksichtigt sein könnten, jede Schlußfolgerung illusorisch machen müsse. Von vorne herein ist dieser Einwand durchaus ernst zu nehmen. Wenn man ihn aber berücksichtigt, so würde es nicht genügen, jede Art im Lande selbst an einem Standort zu studieren, sondern man müßte Material von den verschiedensten Teilen im Lande untersuchen. Dadurch wären die Voruntersuchungen so außerordentlich umfangreich, $\mathrm{da} B$ man sich in absehbarer Zeit auf solche Areale beschränken müßte, die nur eine relativ geringe Artenzahl besitzen. Dies haben als Einzige A. u. D. LövE (1954 in litt.) für Island tatsächlich durchgeführt. Aber gerade hier hat sich gezeigt, daß die Zahlen fast stets mit denen übereinstimmen, die aus anderen Gebieten gewonnen sind. Im Durchschnitt dürfen wir nach meinen Erfahrungen damit rechnen, daß die Zahlen "ohne Überraschungen" bis zu $90 \%$ gesichert sind. Es ist aber notwendig, von Zeit zu Zeit eine Art Inventar aufzunehmen, um zu sehen, inwieweit neue Chromosomenbestimmungen den Prozentsatz der Polyploiden in dem betreffenden Lande verändert haben.

Ehe wir im nachfolgenden selbst dazu übergehen, scheint es mir notwendig zu sein, noch einige Fehlerquellen zu erörtern. In den verschiedenen Floren, die als Grundlage der Artenzahl dienen, wird die Auffassung, ob eine bestimmte Species wirklich eingebürgert und damit zu berücksichtigen ist oder nur als adventive außerhalb der Zählungen zu gelten hat, nicht gleichmäßig vorgenommen. Ferner wird der Umfang einer Species durchaus verschieden gewertet, der Eine spricht schon von guten Arten, wo der Andere nur Subspecies order gar Varietäten sieht. Das gilt besonders für Gattungen, die reich an Apomikten sind, wie bei Alchemilla, Taraxacum und Hieracium. Ich habe hier stets etwa im Sinne von MANSFELD (1940) gewertet. Aber darüber hinaus konnte ich es nicht verantworten, die zahlreichen Kleinarten der LiNNÉschen Sammelart Rubus fruticosus, die fast sämtlich polyploid sind, als Sonderarten zu betrachten, weil dadurch von vornherein in Central- und Nordeuropa der Prozentsatz der Polyploidie stark in die Höhe schnellen würde. Im Gegensatz zu meiner Zählung 1950 habe ich jetzt, wie schon 1934, sie als eine Großart zusammengefaßt, un so mehr als auch neuere Systematiker wie TuRRILL (1936) oder CHRISTIANSEN (1953) sich recht skeptisch zur Gleichwertigkeit der zahlreichen Kleinarten von Rubus mit anderen guten Species stellen. 
Das schwierigste Problem ist aber jedenfalls in einer Reihe von Fällen eine Einigung über die als diploide anzunehmende Basiszahl herzustellen. Da mir hier eine restlose Einigung ausgeschlossen erscheint, möchte ich nicht nur betonen, daß vergleichende Studien über den Polyploidiegrad nicht nur von einem und demselben Autor vorgenommen werden dürfen, sondern auch selbst die Grundsätze angeben, denen ich folge und auf die Ausnahmen hinweisen, die ich davon aus praktischen Gründen vorzunehmen mich genötigt sehe.

Von vorneherein müssen wir uns darüber klar sein, daß wir stets nur die "sekundären Basiszahlen" (TISCHLER, 1954) einer Gattung oder Familie als Ausgangspunkt verwenden, und nicht den Versuch machen dürfen, zu den "primären" vorzudringen, die vielleicht in keiner lebenden Art mehr existieren. Damit wird die Relativität des Diploidiecharakters von vorneherein stark unterstrichen.

Als Beispiel wähle ich die Solanaceen, für die MünTzING (1933), GooDSPEED (1947) u. a. mit guten Gründen $x=6$ annehmen, für die hingegen eine Art mit $n=6$ unbekannt ist. Niemand wird ernstlich alle die Dutzende von Solanum-Species mit $n=12$ als tetraploid bezeichnen. Ferner kennen wir Familien oder Unterfamilien mit solch hohen Chromosomenzahlen, daß wir diese ohne weiteres auf Amphidiploidie zurückführen werden. Trotzdem rechnen wir die Magnoliaceen oder Salicaceen mit $n=19$ als diploid und ebenso beurteilen wir $n=17$ für die Pomoideen, $n=23$ für die Oleaceen, $n=$ 14 für die Betuleen, $n=12$ für die Genisteen. Für die drei letztgenannten Unterfamilien gruppiere ich also nach reichlicher Überlegung anders als 1950. Ähnlich möchte ich jetzt für Geranium noch die Zahlen 9 bis 16 als diploid ansehen, da eine Cäsur bei $12 \mathrm{mir}$ zu künstlich erscheint, während ich für Crassulaceen und Saxifragaceen noch bei meiner Einstufung von 1950 bleibe. Jedenfalls geht es nicht an, wozu manche Autoren geneigt sind, Zahlen, welche durch 2 teilbar sind, als polyploid zu betrachten und solche, die 1 Chromosom mehr oder weniger besitzen, diploid zu nennen. Immer wieder sollte man die Zahlen der Gesamtfamilie oder Unterfamilie zu Rate ziehen. Denn wir dürfen doch nicht vergessen, daß bei der Evolution nicht nur ein Aufsteigen, sondern auch ein Absteigen der Zahlen vorkommt. Das letztere ist sicherlich viel öfter als manche glauben. Seit BABCOCK (1947) und seine Schule zeigten, da $\beta$ in der Gattung Crepis die Arten mit $n=3$ sekundär sich aus denen mit $n=6,5$ oder 4 entwickelt haben, ist es unmöglich, etwa Crepis virens $(n=3)$ als diploid, $C r$. paludosa $(n=6)$ als tetraploid zu werten. Bei den Gramineen, deren Basiszahl in vielen Unterfamilien einwandfrei $b=7$ ist, kennen wir jetzt schon eine ganze Reihe von Arten, die im Gegensatz zu ihren Verwandten auf $n=5$ oder 4 herabgestiegen sind. So möchte ich auch nach wie vor bei Luzula die Reihe $n=6,12,18,24$ als primär betrachten, trotzdem $n=3$ bei $L$. purpurea gefunden wurde (MALHEIROS u. DE CASTRo, 1947). Wenn A. Löve (1954) jetzt in der Caryophyllaceen-Gattung Sagina 
die Reihe 11, 22, 44 aufgedeckt hat, die aufs beste mit den Zahlen der Nachbargattungen harmoniert (Spergula, Spergularia u. Cerastium $b=9$, Arenaria $b=10$, Minuartia $b=12-13$, Moehringia $b=12$, Stellaria $b=11-13$ ), so werden wir die beiden bekannten Sagina-Species mit $n=6$ besser als abgeleitet ansehen, als daß wir alle übrigen für polyploid halten.

Wir können stets aus neuen Untersuchungen überraschend neue Ausblicke erwarten, für die ich freilich bezüglich der in unseren Gebieten behandelten Gattungen nur sehr wenige Beispiele kenne. So nahm man allgemein für Potamogeton $(n=13,26,39)$ an, da $b=13$ wäre; seit aber HARAdA (1942) in Japan auch $n=14$ und 21 feststellte, liegt es näher, alle Zahlen auf $b=7$ zurückzuführen, um so mehr, als die Zahlen 6 und 7 bei den Helobiern eine Hauptrolle spielen. Z $Z$ weitens kannten wir bis vor kurzem für Orobanche nur Arten mit $n=19$. Aber ŽUKov (1939) und GARDÉ (1951) wiesen auch solche mit $n=12$ nach, und JENSEN (1951) fand eine Species mit $n=18$. Auch hier liegt eine Zurückführung auf $b=6$ doch sehr nahe'. Drittens betrachte ich die etwas ungewöhnliche Zahl von $n=-17$ bei der CaryophyllaceenGattung Gypsophila jetzt als hypotetraploid, nachdem FAVARGER (1946) in G. libanotica $n=9$ aufdeckte.

Gerade innerhalb einer Gattung hat sich neben der typischen Allotetraploidie Stabilisierung auf amphidiploid entstandener Alloaneuploidie anscheinend öfter herausgebildet. Bei Berücksichtigung der Nachbargattungen werden wir klarer sehen, wie hier zu gruppieren ist. Als Beispiel nenne ich die seit langem studierte Gattung Campanula (mit $n=8,9$ u. 10, daneben $n=17$ ); nur die drei erstgenannten Zahlen werte ich als diploid. Sehr merkwürdig war in dieser Hinsicht bis vor kurzem die Lage für die Gattung Cirsium, für das wir nur $n=17,34$ etc. fanden. Die Nachbargattung Carduus, die anfangs mit Cirsizum in einer Gattung vereinigt war, besitzt $n=8$ u. 11 , und bei Centaurea zählen wir $b=8-12$. Jetzt hat OwNBEY (1951) aber auch bei Cirsium Arten mit $n=10$ gefunden und daher werte ich die Zahl 17 nicht mehr als diploid.

Alle derartigen Gattungen spielen innerhalb der Flora eines ganzen Landes eine so unbedeutende Rolle, daß der Prozentsatz an Polyploiden bei anderer Wertung kaum signifikant verschoben würde. Nur in drei Fällen kann ich mich aus praktischen Gründen nicht dazu verstehen, das Prinzip der Basiszahl einer Gattung "revolutionär” zur Geltung kommen zu lassen, weil dadurch stärkere Störungen bei den Berechnungen nicht zu vermeiden wären. Da aber in sämtlichen Ländern der Diploidiegrad gleichmäßig angenommen wird, würden wir in dieselbe Lage kommen, wenn wir zufällig, um bei unserem obigen Beispiel zu bleiben, eine Solanacee mit $n=6$ entdeckten. Ich glaube kaum, daß man hier von der konservativen Beurteilung abgehen würde.

1 Interessant ist es, daß auch Lathraea squamaria $n=18$ (v. Witsch, 1942) oder 21 (Gates u. LatTere, 1927) besitzt. Tarnavschi (1948) sowie Claphaim, Tutin u. Warburg (1952) sind jedenfalls so ketzerisch, die Gattung von den Scrophulariaceen zu entfernen und mit den Orobanchaceen zu vereinigen, 
Die drei Beispiele sind die folgenden: Bei den Urticaceen kennen wir zur Zeit für 39 Species die Grundzahlen 12 bis 14 . Diese schließen sich eng an die der verwandten Familien Ulmaceen und Moraceen an. Nur Helxine Soleirolii, zwei der drei Cannabaceen und einige Celtis-Species sind auf $n=$ 10 gegangen und Humulus japonicus gar auf $n=8$. Das Auf- und Absteigen innerhalb der Moraceen-Gattung Dorstenia $(b=14)$ hat mein Schüler KRAUSE (1930) gut verfolgt. Der gleiche Autor fand nun aber in Parietaria officinalis $n=7$ (mit regulärer Meiose), während $P$. judalica wieder $n=13$ besitzt.

Das zweite Beispiel haben wir bei den Orchideen. Alle einheimischen Monandrae besitzen Zahlen von $n=14-21$, nur bei Epipactis latifolia hat kürzlich WEIJER (1952) neben $n=20$ auch eine Rasse mit $n=10$ entdeckt. Und das dritte Beispiel bezieht sich paradoxerweise gerade auf Mitglieder der Ericales von denen wir oben bei unseren Betrachtungen ausgingen. Für die Ericaceen, Empetraceen und Pirolaceen gelten eindeutig die Zahlen $n=12$ und 13, seltener auch $n=11$. In mehr als 150 Species sind diese erhärtet. Lediglich Calluna vulgaris und Monotropa hypopithys subsp. hypophega besitzen $n=8$. In den verwandten Familien der Diapensiaceen treffen wir öfter auch $n=6$, der Clethraceen $n=8$, der Epacridaceen auBerdem $n=4,7$, 8,9 und wieder 12 u. 13 an. Die Systematiker müssen entscheiden, ob bei diesen Arten mehr ursprüngliche als abgeleitete morphologische Merkmale zu beobachten sind. ENGLER-DiELs (1936) führen die Diapensiaceen und Clethraceen vor den Ericaceen, die Epacridaceen hinter ihnen auf.

Sollen wir jetzt fast alle Vertreter der eben genannten drei Gruppen als polyploid werten? Meine Forderung nach dem gleichen Autor für vergleichende Polyploidieschätzung wird immerhin durch derartige Beispiele unterstrichen.

Damit können wir uns zu den Untersuchungen über das Vorkommen von Polyploiden in den verschiedenen Arealen Europas wenden. Als Beispiel für eines, das im Mittelmeergebiet besonders wenig extreme Verhältnisse bietet, hatte ich schon 1946 die Inselgruppe der Kykladen ausgewählt. Durch RECHINGERs (1944) Studien wissen wir sehr gut über die Flora Bescheid. Und der Wiener Kollege hat jetzt meine aus seinen Arbeiten ausgezogene Liste freundlicherweise noch durchgesehen und ergänzt. Die Insel Sizilien, die ich 1934 als Beispiel nahm, ist orographisch weniger einheitlich und ihre Flora mir weniger genau bekannt. Als $Z$ wischenstation nach Centraleuropa wählte ich sodann die Listen von Rumänien, die TARNAvschi (1948) schon vergleichend chromosomal behandelte, und Ungarn, für das wir in der vorzüglichen Flora von v. Soó (1951) eine Basis besitzen. Nach Norden habe ich einmal, den Listen von A.u. D. LövE (1948) folgend, Schweden und die Faröer gewählt sowie Groß-Britannien nach der Flora von CLAPHAM, Tutin u. WARBURg (1952) untersucht. Für Island gab A. Löve sehr zuvorkommend mir brieflich das gesamte von ihm studierte Material 1954. SüdwestGrönland studierte ich nach PoLUNIN (1942), und für Spitzbergen hatte Kollege 
SUESSENGUTh-München die Liebenswürdigkeit, mir ein Verzeichnis der dort wachsenden Arten zu schicken, das ich nur leicht zu verändern brauchte. Alle diese verschiedenen Floren wurden zu der von Centraleuropa in Beziehung gesetzt, und es ergab sich, wie wir sehen werden, dabei eine ganz klare Beziehung im Sinne der ursprünglichen Idee HAGERUPs.

Ausgangspunkt war Centraleuropa, das ich selbst bereits 1950 behandelt habe. Nach Weglassung der Rubus-Kleinarten und nach Einfügung leichter sonstiger Veränderungen zähle ich hier jetzt 2287 Dikotyle, 622 Monokytyle, also 2909 Angiospermen, die zu 79,3\%, 82\% und 79,9\% chromosomal untersucht sind. Ich erhalte:

\begin{tabular}{ccccccccc} 
& Dikotyle & \multicolumn{3}{c}{ Monokotyle } & \multicolumn{3}{c}{ Angiospermen } \\
Dipl. & Polpl. & D + P & Dipl. & Polpl. & D + P & Dipl. & Polpl. & D + P \\
875 & 784 & 155 & 163 & 292 & 55 & 1038 & 1076 & 210 \\
$=48,2 \%$ & $=43,2 \%$ & $8,6 \%$ & $=32 \%$ & $=57,3 \%$ & $=10,7 \%$ & $=44,7 \%$ & $=46,3 \%$ & $=9 \%$
\end{tabular}

Um einen genauen Vergleich mit meinen Zählungen von $1950 \mathrm{zu}$ ermöglichen, füge ich hinzu, daB mit EinschluB der untersuchten Rubi fruticosi als Sonderarten das Verhältnis für die Angiospermen ist:

$\begin{array}{rrrr}\text { Chrom. bek. } & \text { Dipl. } & \text { Polpl. } & \text { D+P } \\ \text { (1954) } 79,6 \% & 43,5 \% & 47,7 \% & 8,8 \% \\ (1950) 73,7 \% & 43 \% & 43,3 \% & 8,7 \%\end{array}$

Lasse ich vorläufig die Arten mit diploiden und polyploiden Rassen als " ununtersucht" aus (die Angiospermen wären dann jetzt zu $72,7 \%$ untersucht) ergibt das:

\begin{tabular}{lccccc}
\multicolumn{2}{c}{ Dikotyle } & \multicolumn{2}{c}{ Monokotyle } & \multicolumn{2}{c}{ Angiospermen } \\
Dipl. & Polpl. & Dipl. & Polpl. & Dipl. & Polpl. \\
875 & 784 & 163 & 191 & 1038 & 1076 \\
$=52,7 \%$ & $=47,3 \%$ & $=35,8 \%$ & $=64,2 \%$ & $=49,1 \%$ & $=50,9 \%$
\end{tabular}

\section{Kykladen}

Ich zähle hier 938 Dikotyle, 248 Monokotyle, somit 1186 Angiospermen. Von diesen sind erst $52,3 \%, 58,1 \%$ und $53,5 \%$ chromosomal studiert. Die Prozentzahlen sind für

$$
\begin{array}{ccccccccc} 
& \text { Dikotyle } & \multicolumn{3}{c}{\text { Monokotyle }} & \multicolumn{3}{c}{\text { Angiospermen }} \\
\text { Dipl. } & \text { Polpl. } & \text { D }+ \text { P } & \text { Dipl. } & \text { Polpl. } & \text { D }+ \text { P } & \text { Dipl. } & \text { Polpl. } & \text { D +P } \\
306 & 147 & 38 & 59 & 67 & 18 & 365 & 214 & 56 \\
=62,3 \% & =30 \% & =7,7 \% & =41 \% & =46,5 \% & =12,5 \% & =57,5 \% & =33,7 \% & =8,8 \%
\end{array}
$$

Unter Weglassung der Arten mit diploiden und polyploiden Rassen bleiben $579=48,8 \%$ chromosomal untersuchte Species.

\begin{tabular}{cccccc} 
Dipl. & Polpl. & Dipl. & Polpl. & Dipl. & Polpl. \\
306 & 147 & 59 & 67 & 365 & 214 \\
$=67,6 \%$ & $=32,4 \%$ & $=46,8 \%$ & $=53,2 \%$ & $=63 \%$ & $=37 \%$ \\
\hline
\end{tabular}

Es ist von sehr großem Interesse, daß jüngst DE MESQuita RoDigues (1953) wenigstens die Florula eines kleinen Gebietes im westmediterranen 
Gebiet, nämlich in Portugal, studiert hat. Es handelt sich dabei um eine Strandflora am Mündungsausgang des Rio Mondega unweit von Coimbra in Größe von ca. $20 \mathrm{qkm}$. Von den hier beobachteten 174 Angiospermen wurden vom Verf. selbst 104 auf ihre Chromosomenzahlen untersucht, die übrigen nach der vorhandenen Literatur angegeben:

$\begin{array}{lrccc} & & \text { Dipl. } & \text { Polpl. } & \text { D }+ \text { P } \\ \text { Dikotyle } & 126 & 84(=66,7 \%) & 37(=29,4 \%) & 5(=3,9 \%) \\ \text { Monokotyle } & 48 & 16(=33,3 \%) & 30(=62,5 \%) & 2(=4,2 \%) \\ \text { Angiosp. } & 174 & 100(=57,5 \%) & 67(=38,5 \%) & 7(=4 \%)\end{array}$

Läßt man die Arten mit $D+P$ fort, erhält man

\begin{tabular}{lrll} 
Dikotyle & 121 & Dipl. & Polpl. \\
Monokotyle & 46 & $69,4 \%$ & $\underline{30,6 \%}$ \\
Angiosp. & 167 & $60 \%, 8 \%$ & $\underline{65 \%}, 2 \%$ \\
\hline
\end{tabular}

Der Polyploidiegrad dieser Strandflora ist gegenüber dem der Gesamtangiospermenflora auf den Kykladen signifikant nicht verändert.

\section{Rumänien}

Vorhanden sind nach Weglassung der systematischen Einheiten, die TARNAVSCHI (1948) nur als Subspecies wertet, 2691 Dikotyle, 674 Monokotyle, also 3365 Angiospermen. Chromosomal untersucht sind davon 65,3\%, 74,3\% und $67,1 \%$, es betragen

\begin{tabular}{ccccccccc} 
& Dikotyle & \multicolumn{3}{c}{ Monokotyle } & \multicolumn{3}{c}{ Angiospermen } \\
Dipl. & Polpl. & D $+\mathrm{P}$ & Dipl. & Polpl. & D + P & Dipl. & Polpl. & $D+P$ \\
946 & 698 & 113 & 171 & 284 & 46 & 1117 & 982 & 159 \\
$=53,8 \%$ & $=39,8 \%$ & $=6,4 \%$ & $=34,2 \%$ & $=56,7 \%$ & $=9,1 \%$ & $=49,5 \%$ & $=43,5 \%$ & $7 \%$
\end{tabular}

Bei Weglassung der Arten mit diploiden und polyploiden Rassen bleiben 2099 Species $=65,3 \%$ chromosomal untersuchte.

\begin{tabular}{cccccc}
\multicolumn{2}{c}{ Dikotyle } & \multicolumn{2}{c}{ Monokotyle } & \multicolumn{2}{c}{ Angiospermen } \\
Dipl. & Polpl. & Dipl. & Polpl. & Dipl. & Polpl. \\
946 & 698 & 171 & 284 & 117 & 982 \\
$=57,6 \%$ & $=42,4 \%$ & $=37,6 \%$ & $=62,4 \%$ & $=53,2 \%$ & $=46,8 \%$ \\
\hline
\end{tabular}

TARNAVSCHI hat sämtliche Arten oder Unterarten mit diploiden und polyploiden Rassen ohne weiteres den Polyploiden zugezählt. So erhielt er $52,4 \%$ diploide und 47,34\% polyploide Angiospermen. Zählte er sämtliche "Einheiten" gesondert, ergaben sich 59,42\% Diploide und 40,58\% Polyploide. Er selbst hat eine größere Reihe von Arten, neuerdings 1948 noch 115 Species, in Rumänien chromosomal untersucht.

\section{Ungarn}

Hier zähle ich nach dem Studium der Flora von v. Soó (1951) 1610 Dikotyle, 429 Monokotyle, also 2039 Angiospermen, die zu 77\%, 84,8\% und $78,7 \%$ chromosomal untersucht sind. Es betragen 


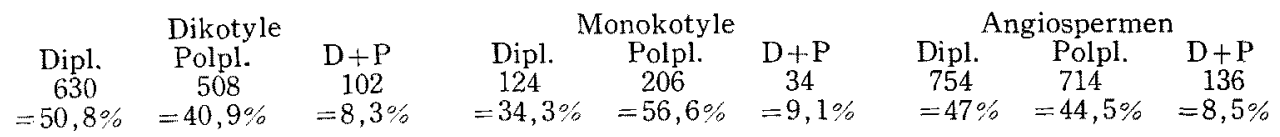

Nach Weglassung der Arten mit diploiden und polyploiden Rassen bleiben 1468 Species, also $72 \%$ chromosomal untersuchte.

\begin{tabular}{cccccc}
\multicolumn{2}{c}{ Dikotyle } & \multicolumn{2}{c}{ Monokotyle } & \multicolumn{2}{c}{ Angiospermen } \\
Dipl. & Polpl. & Dipl. & Polpl. & Dipl. & Polpl. \\
630 & 508 & 124 & 206 & 754 & 714 \\
$=55,4 \%$ & $=44,6 \%$ & $=37,6 \%$ & $=62,4 \%$ & $=51,4 \%$ & $=48,6 \%$
\end{tabular}

Ungarn hat eine viel gleichmäßigere Flora als das orographisch stark gegliederte Rumänien. Darum ist in Ungarn die Artenzahl viel geringer. FELFöLDY (1948) und Pólya $(1948,1949)$ untersuchten eine ganze Reihe von Species im Lande selbst.

Wenden wir uns jetzt den Floren des Nordens zu, so mag die von

\section{Schleswig-Holstein}

deshalb besonders behandelt werden, nicht nur weil ich sie zuerst analysierte, sondern auch weil sie als die nördlichste in Centraleuropa ein besonders starkes Florengefälle aufweist (ChRISTIANSEN, 1930) und damit eine vorzügliche "Brücke zum Norden" darstellt. Als ich mit meinen Berechnungen begann, waren erst 69,4\% der Dikotylen, 59,7\% der Monokotylen, 66,7\% der Angiospermen chromosomal untersucht. Heute sind es $97 \%$ Dikotyle, 94,9\% Monokotyle und 96,4\% Angiospermen. Als Grundlage diente dabei die neuerschienene sehr sorgfältige Zusammenstellung in der Flora von CHRIsTiansen (1953). Ich zähle danach 789 Dikotyle, 292 Monokotyle, also 1081 Angiospermen. Chromosomal sind davon $1042=96,4 \%$ untersucht.

\begin{tabular}{|c|c|c|c|c|c|c|c|}
\hline \multicolumn{3}{|c|}{ Dikotyle } & \multicolumn{3}{|c|}{ Monokotyle } & \multicolumn{2}{|c|}{ Angiospermen } \\
\hline$D$ & $\begin{array}{c}\text { Polpl. } \\
341 \\
=44,6 \%\end{array}$ & $\begin{array}{l}D+P \\
=56 \\
=7,3 \%\end{array}$ & $\begin{aligned} & \text { Dipl. } \\
& 70 \\
= & 25,3 \%\end{aligned}$ & $\begin{array}{c}\text { Polpl. } \\
184 \\
=66,4 \%\end{array}$ & $\begin{aligned} & D+P \\
& 23 \\
= & 8,3 \%\end{aligned}$ & $\begin{array}{l}\text { Dipl. } \\
438 \\
=42 \%\end{array}$ & $\begin{array}{cc}\text { Polpl. } & \mathrm{D}+7 \\
525 & 7 \\
=50,4 \% & =7\end{array}$ \\
\hline
\end{tabular}

Und wieder unter Fortlassung der Arten mit diploiden und polyploiden Rassen, wobei $963=89,1 \%$ chromosomal untersuchte Arten übrig bleiben

\begin{tabular}{cccccc}
\multicolumn{2}{c}{ Dikotyle } & \multicolumn{2}{c}{ Monokotyle } & \multicolumn{2}{c}{ Angiospermen } \\
Dipl. & Polpl. & Dipl. & Polpl. & Dipl. & Polpl. \\
368 & 341 & 70 & 184 & 438 & 525 \\
$=51,9 \%$ & $=48,1 \%$ & $=27,6 \%$ & $=72,4 \%$ & $=45,5 \%$ & $=54,5 \%$
\end{tabular}

Der Polyploidiegehalt ist also wesentlich höher, als ich ihn 1934 angab. Das hängt aber nur zum Teil von den inzwischen neu untersuchten Arten ab. Denn ich habe vor 2 Jahrzehnten nur "Mindestzahlen" an Polyploiden angegeben, und viele Arten überhaupt nicht zu werten versucht. Auf Grund meiner jetzigen Kenntnisse würde ich das damals vorliegende Material beträchtlich anders beurteilen. An Polyploiden rechne ich

\begin{tabular}{lccc}
\multicolumn{2}{c}{1934} & & \multicolumn{2}{c}{1954} \\
Dikotyle & $210=39 \%$ & Dikotyle & $235=43 \%$ \\
Monokotyle & $105=60 \%$ & Monokotyle & $111=63,4 \%$ \\
Angiospermen & $315=44,1 \%$ & Angiospermen & $346=48,5 \%$
\end{tabular}


Der tatsächliche Polyploidiegrad ist somit nicht um $10,4 \%$, sondern nur um $6 \%$ gestiegen.

\section{Schweden}

In dem Chromosomenkatalog von A. u. D. Löve (1948) besitzen wir eine schöne Zusammenstellung der für Skandinavien untersuchten Arten. Ich zähle hier 1091 Dikotyle, 435 Monokotyle. also 1526 Angiospermen ${ }^{1}$. Chromosomal untersucht sind davon $1023(=93,8 \%)$ Dikotyle, 404=92,8\% Monokotyle, also $1427(=93,5 \%)$ Angiospermen.

\begin{tabular}{ccccccccc} 
& Dikotyle & \multicolumn{3}{c}{ Monokotyle } & \multicolumn{3}{c}{ Angiospermen } \\
Dipl. & Polpl. & D $+\mathrm{P}$ & Dipl. & Polpl. & D + P & Dipl. & Polpl. & D + P \\
465 & 479 & 79 & 103 & 274 & 27 & 568 & 753 & 106 \\
$=45,6 \%$ & $=46,8 \%$ & $=7,6 \%$ & $=25,5 \%$ & $-67,8 \%$ & $=6,7 \%$ & $=39,8 \%$ & $=52,8 \%$ & $=7,4 \%$
\end{tabular}

Und nach Weglassung der Arten mit diploiden und polyploiden Rassen bleiben 1123 das heißt $86,6 \%$ chromosomal untersuchte.

\begin{tabular}{cccccc}
\multicolumn{2}{c}{ Dikotyle } & \multicolumn{2}{c}{ Monokotyle } & \multicolumn{2}{c}{ Angiospermen } \\
Dipl. & Polpl. & Dipl. & Polpl. & Dipl. & Polpl. \\
465 & 479 & 103 & 274 & 568 & 753 \\
$=50,7 \%$ & $=49,3 \%$ & $=27,3 \%$ & $=72,1 \%$ & $=43,1 \%$ & $=56,9 \%$
\end{tabular}

Da sich für Centraleuropa ein starkes Gefälle gegen Norden hin bemerkbar machte, wollte ich auch hier die Flora von Norrland gesondert betrachten. Der Unterschied gegenüber dem Gesamtland war indes nur unerheblich. Ich zähle dabei 956 Arten, von denen 94,1\% chromosomal untersucht sind.

\begin{tabular}{ccccccccc} 
& Dikotyle & \multicolumn{4}{c}{ Monokotyle } & \multicolumn{3}{c}{ Angiospermen } \\
Dipl. & Polpl. & D + P & Dipl. & Polpl. & D + P & Dipl. & Polpl. & D +P \\
288 & 301 & 49 & 56 & 198 & 17 & 344 & 499 & 66 \\
$=45,3 \%$ & $=47,2 \%$ & $=7,5 \%$ & $=20,7 \%$ & $=73,1 \%$ & $=6,2 \%$ & $=38 \%$ & $=54,9 \%$ & $=7,1 \%$
\end{tabular}

Bei Weglassung der Arten mit diploiden und polyploiden Rassen bleiben $844=88,2 \%$ chromosomal untersuchte.

\begin{tabular}{cccccc}
\multicolumn{2}{c}{ Dikotyle } & \multicolumn{2}{c}{ Monokotyle } & \multicolumn{2}{c}{ Angiospermen } \\
Dipl. & Polpl. & Dipl. & Polpl. & Dipl. & Polpl. \\
288 & 301 & 56 & 198 & 344 & 499 \\
$=48,9 \%$ & $=51,1 \%$ & $=22 \%$ & $=78 \%$ & $=40,8 \%$ & $=59,2 \%$.
\end{tabular}

A. u. D. Löve (1949) haben auf Grund der damals vorliegenden Zahlen für die skandinavischen Länder, wie folgt, gezählt. Ich berichte dabei nur die Zahlen für die Gesamtangiospermen. Sie erhielten für Dänemark 53,5\%, Schweden 56,0\%, Finnland 57,3\%, Norwegen 57,6\%. Die Unterschiede sind nicht signifikant. Ich habe mich daraufhin, da die weitaus größte Zahl der Arten gemeinsam ist, auf die soeben mitgeteilte Flora von Schweden beschränkt.

1 Löve (1948) rechnet mit 1582 Arten (incl. der zahlreichen Rubus-Kleinarten,) 1949 sogar mit 1645 Arten. 


\section{Groß-Britannien}

A. u. D. Löve (1949) berechneten hier auf Grund der ihnen bekannten Chromosomenzahlen den Polyploidiegrad für die von ihnen zu 1630 angenommenen Angiospermen auf 56,7\%, dabei 49,8\% für die Dikotylen und 75,1\% für die Monokotylen. HASKELL (1952) zählte nur 1509 Angiospermen, dabej 1092 Dikotyle, 417 Monokotyle. Er erhielt:

\begin{tabular}{ccccccccc} 
& Dikotyle & \multicolumn{3}{c}{ Monokotyle } & \multicolumn{3}{c}{ Angiospermen } \\
Dipl. & Polpl. & $\mathrm{D}+\mathrm{P}$ & Dipl. & Polpl. & $\mathrm{D}+\mathrm{P}$ & Dipl. & Polpl. & $\mathrm{D}+\mathrm{P}$ \\
511 & 435 & 38 & 91 & 184 & 17 & 602 & 619 & 55 \\
$=52 \%$ & $=44,2 \%$ & $=3,8 \%$ & $=31,2 \%$ & $=63 \%$ & $=5,8 \%$ & $=47,2 \%$ & $=48,5 \%$ & $=4,3 \%$
\end{tabular}

Lassen wir die Arten mit diploiden und polyploiden Rassen fort, ergibt das für

\begin{tabular}{cccccc}
\multicolumn{2}{c}{ Dikotyle } & \multicolumn{2}{c}{ Monokotyle } & \multicolumn{2}{c}{ Angiospermen } \\
Dipl. & Polpl. & Dipl. & Polpl. & Dipl. & Polpl. \\
511 & 435 & 91 & 184 & 602 & 619 \\
$=54,2 \%$ & $=45,8 \%$ & $=33,1 \%$ & $=66,9 \%$ & $=49,2 \%$ & $=50,8 \%$
\end{tabular}

Nach der Flora von Clapham, Tutin u. WARBurg (1952) zähle ich dagegen, sofern ich die als "naturalized" und "stabilized" zu der ursprünglichen Flora dazugekommenen Arten mitrechne, an Dikotylen 1328, Monokotylen 450, Angiospermen 1778. Davon sind chromosomal untersucht 1141 (=85,9\%), $408(=90,7 \%) 1549(=87,1 \%)$.

\begin{tabular}{ccccccccc} 
& Dikotyle & \multicolumn{3}{c}{ Monokotyle } & \multicolumn{3}{c}{ Angiospermen } \\
Dipl. & Polpl. & D + P & Dipl. & Polpl. & D + P & Dipl. & Polpl. & D + P \\
553 & 508 & 80 & 118 & 257 & 33 & 671 & 765 & 113 \\
$=48,5 \%$ & $=44,5 \%$ & $=7 \%$ & $=28,9 \%$ & $=63 \%$ & $=8,1 \%$ & $=43,3 \%$ & $=49,4 \%$ & $=7,3 \%$
\end{tabular}

Lasse ich wieder die Arten mit diploiden und polyploiden Rassen fort, bleiben 1436 Species, also 80,7\% der Gesamtzahl.

\begin{tabular}{cccccc}
\multicolumn{2}{c}{ Dikotyle } & \multicolumn{2}{c}{ Monokotyle } & \multicolumn{2}{c}{ Angiospermen } \\
Dipl. & Polpl. & Dipl. & Polpl. & Dipl. & Polpl. \\
553 & 508 & 118 & 257 & 671 & 765 \\
$=52,1 \%$ & $=47,9 \%$ & $=31,5 \%$ & $=68,5 \%$ & $=46,7 \%$ & $=53,3 \%$
\end{tabular}

Die Unterschiede in der Berechnung der Polyploiden zwischen A. u. D. Löve, HASKELL und mir beruhen somit wohl in erster Linie auf der verschiedenen Zahl der als eingebürgert angesehenen Arten.

\section{Faröer}

Bereits 1934 hatte ich die Flora dieser Inselgruppe bei meinen Berechnungen berücksichtigt. Sie war damals auf 172 Dikotyle und 95 Monokotyle, also auf 267 Angiospermen angenommen, von denen bei $162(=60,4 \%)$ chromosomale Bestimmungen vorlagen. Als Mindestsatz nahm ich 49,4\% Polyploide an. Jetzt zähle ich nach der Flora von A.u. D. Löve (1948) 298 
Angiospermen, 191 Dikotyle, 107 Monokotyle, für die 289, also 97\% Chromosomenzahlen vorliegen.

$$
\begin{array}{ccccccccc} 
& \text { Dikotyle } & \multicolumn{3}{c}{\text { Monokotyle }} & \multicolumn{3}{c}{\text { Angiospermen }} \\
\text { Dipl. } & \text { Polpl. } & \text { D }+\mathrm{P} & \text { Dipl. } & \text { Polpl. } & \text { D }+ \text { P } & \text { Dipl. } & \text { Polpl. } & \mathrm{D}+\mathrm{P} \\
68 & 99 & 17 & 16 & 82 & 7 & 84 & 181 & 24 \\
=37 \% & =53,8 \% & =9,2 \% & =15,2 \% & =78,1 \% & =6,7 \% & =20,1 \% & =62,6 \% & =8,3 \%
\end{array}
$$

Unter Weglassung der Arten mit diploiden und polyploiden Rassen bleiben 265 Arten $=88,9 \%$ der Gesamtzahl.

$$
\begin{array}{cccccc}
\multicolumn{2}{c}{\text { Dikotyle }} & \multicolumn{2}{c}{\text { Monokotyle }} & \multicolumn{2}{c}{\text { Angiospermen }} \\
\text { Dipl. } & \text { Polpl. } & \text { Dipl. } & \text { Polpl. } & \text { Dipl. } & \text { Polpt } \\
68 & 99 & 16 & 81 & 84 & 180 \\
=40,7 \% & =59,3 \% & =16,5 \% & =83,5 \% & =31,7 \% & =68,3
\end{array}
$$

$\begin{array}{cc}\text { Dipl. } & \text { Polpl. } \\ 84 & 180 \\ =31,7 \% & =68,3 \%\end{array}$

Hier liegt die einzige stärkere Diskrepanz mit A. u. D. Löve vor. In ihrer Zusammenstellung (1949) zählten sie nur 61,3\% Polyploide für die Angiospermen. Der Unterschied dürfte dadurch zu erklären sein, daß sie von 324 Angiospermen ausgehen. Ich vermute, daß bei diesen 26 zusätzlichen Arten, die sich wohl auf neuerlich eingebürgerte beziehen, sich eine größere Zahl Diploider befindet.

\begin{tabular}{|c|c|c|c|c|c|c|c|c|}
\hline \multicolumn{3}{|c|}{ Dikotyle } & \multicolumn{3}{|c|}{ Monokotyle } & \multicolumn{3}{|c|}{ Angiospermen } \\
\hline & & ach Löve & & & ch Löve & & & ch Löve \\
\hline $\begin{array}{r}\text { Dipl. } \\
149\end{array}$ & $\begin{array}{c}\text { Polpl. } \\
196\end{array}$ & Polpl. & $\begin{array}{c}\text { Dipl. } \\
22\end{array}$ & $\begin{array}{c}\text { Polpl. } \\
135\end{array}$ & Polpl. & $\begin{array}{r}\text { Dipl. } \\
171\end{array}$ & $\begin{array}{c}\text { Polpl. } \\
331\end{array}$ & Polpl. \\
\hline$=43,2 \%$ & $=56,8 \%$ & $=55,3 \%$ & $=14 \%$ & $=86 \%$ & $88,3 \%$ & $=34,1 \%$ & $=65,9 \%$ & $65,9 \%$ \\
\hline
\end{tabular}

\section{Island}

Dank der sehr eingehenden Studien von A.u. D. Löve (1954 in litt.) ist diese Insel das chromosomal am besten untersuchte Land der ganzen Erde. Die beiden Autoren haben die Flora zu 100\% chromosomal studiert. Herr Kollege Löve hatte die überaus große Freundlichkeit, mir seine gesamten noch nicht publizierten Berechnungen zuzusenden. In einigen Fällen weiche ich etwas von der Artabgrenzung sowie in der Beurteilung des Polyploidiegrades ab. Doch hatte das hier einen recht geringen Einfluß auf das Gesamtergebnis. Berücksichtigt man sämtliche jetzt in Island eingebürgerte Arten, so zählt man 345 Dikotyle, 157 Monokotyle, also 502 Angiospermen.

Berücksichtigen wir nur die wirklich einheimische Flora und abstrahieren wir von den vielen nur "eingebürgerten" Species, erhalten wir

\begin{tabular}{ccccccccc} 
& \multicolumn{3}{c}{ Dikotyle } & \multicolumn{3}{c}{ Monokotyle } & \multicolumn{2}{c}{ Angiospermen } \\
& \multicolumn{2}{c}{ nach Löve } & \multicolumn{2}{c}{ nach Löve } & \multicolumn{2}{c}{ nach Löve } \\
Dipl. & Polpl. & Polpl. & Dipl. & Polpl. & Polpl. & Dipl. & Polpl. & Polpl. \\
90 & 154 & - & 18 & 123 & - & 108 & 277 & - \\
$=36,9 \%$ & $=63,1 \%$ & $60,25 \%$ & $=12,8 \%$ & $=87,2 \%$ & $\underline{89,96 \%}=28,1 \%$ & $=71,9 \%$ & $71,2 \%$ \\
\hline
\end{tabular}

Arten mit diploiden und polyploiden Rassen sind nicht aufgeführt resp. sie sind also eigene Species bezeichnet. 


\section{S. W. Gronland}

Zum vergleich mag auch die Flora dieses Areals zwischen $48^{\circ} 10^{\prime}$ bis $45^{\circ} 11^{\prime} \mathrm{W}$ und $61^{\circ} 15^{\prime}$ bis $60^{\circ} 25^{\prime} \mathrm{N}$ herangezogen werden, die PoLuniN (1942) aufführt. Er gibt 157 Dikotyle und 105 Monokotyle, also 262 Angiospermen als ursprünglich an, daneben noch eine Anzahl eingeschleppte, von denen mir noch nicht klar ist, welche man als wirklich eingebürgert betrachten darf. Sondere ich nach Analogie mit Island diejenigen aus, die man dazu vielleicht rechnen kann, ergibt das 181 Dikotyle, 111 Monokotyle, in summa 292 Angiospermen. Chromosomal sind davon $86 \%$ untersucht.

\begin{tabular}{cccccc}
\multicolumn{2}{c}{ Dikotyle } & \multicolumn{2}{c}{ Monokotyle } & \multicolumn{2}{c}{ Angiospermen } \\
Dipl. & Polpl. & Dipl. & Polpl. & Dipl. & Polpl. \\
61 & 95 & 12 & 84 & 73 & 178 \\
$=39,1 \%$ & $=60,9 \%$ & $=12,5 \%$ & $=87,5 \%$ & $=29 \%$ & $=71 \%$ \\
\hline
\end{tabular}

Bei Berücksichtigung nur der indigenen Flora die zu 85,1\% chromosomal bekannt ist, erhalte ich für die 223 Arten

\begin{tabular}{cccccc}
\multicolumn{2}{c}{ Dikotyle } & \multicolumn{2}{c}{ Monokotyle } & \multicolumn{2}{c}{ Angiospermen } \\
Dipl. & Polpl. & Dipl. & Polpl. & Dipl. & Polpl \\
48 & 84 & 10 & 81 & 58 & 165 \\
$=36,4 \%$ & $=63,6 \%$ & $=11 \%$ & $=89 \%$ & $=26 \%$ & $=74 \%$ \\
\hline
\end{tabular}

A. u. D. Love (1949) haben für O. Grönland den Polyploidiegrad bei den Dikotylen mit 60,2\%, den Monokotylen mit 92,7\%, den Angiospermen mit $71,9 \%$ bestimmt.

\section{Spitzbergen}

Als letztes Areal sei diese nördlichste Inselgruppe studiert, für die schon A. u. D. Löve (1949) angeben, daß 82,1\% der 135 Angiospermen cytologische bekannt wären. Ich rechne mit 98 Dikotylen, 47 Monokotylen, also 145 Angiospermen und finde davon 80 Dikotyle $(=81,6 \%), 42$ Monokotyle $(=89,4 \%), 122$ Angiospermen $(=84,1 \%)$ chromosomal untersucht. Diese Studien verdanken wir in erster Linie FLovik $(1928,1940)$.

\begin{tabular}{ccccccccc} 
& \multicolumn{3}{c}{ Dikotyle } & \multicolumn{3}{c}{ Monokotyle } & \multicolumn{2}{c}{ Angiospermen } \\
Dipl. & Polpl. & Polpl. & Dipl. & Polpl. & Polpl. & Dipl. & Polpl. & Polpl. \\
28 & 52 & - & 1 & 41 & - & 29 & 93 & - \\
$=35 \%$ & $=65 \%$ & $61,4 \%$ & $=2,4 \%$ & $=97,6 \%$ & $95 \%$ & $=23,8 \%$ & $=76,2 \%$ & $73,6 \%$
\end{tabular}

Für Island, S. W. Grönland und Spitzbergen haben wir keine Arten mit diploiden und polyploiden Rassen aufgeführt, die wir bei den südlicheren Arealen der Vorsicht Wegen als ununtersucht fortließen. Doch mag es erlaubt sein, für die besser bekannten Floren von Centraleuropa, Schweden und GroßBritannien diese jedes $\mathrm{Mal}$ den diploiden und polyploiden zuzuschlagen. Wir haben Grund zu der Annahme, daß wirklich beide Rassen im Gebiet vorhanden sind. Wo wir wissen, daß nur eine Rasse in einem Ploidiegrad im Gebiet 
vorkommt, haben wir selbstverständlich ohnehin diese von vorneherein bei unseren obigen Berechnungen mitgezählt. Um Beispiele anzugeben, so haben wir Aegopodium podagraria für Centraleuropa und den Norden nur tetraploid genommen, trotzdem wir aus Portugal eine diploide Rasse kennen. Wir haben Galium verum bei den Kykladen diploid und tetraploid, in Central und Nordeuropa nur tetraploid gerechnet. Primula farinosa ist im allgemeinen nur diploid. Lediglich auf der schwedischen Insel Gothland ist sie auch tetraploid bekannt geworden. Darum habe ich sie auch nur für Schweden unter der Gruppe mit diploiden und polyploiden Rassen aufgeführt. Verfahren wir jetzt für die drei eben genannten Areale so, daß wir die strittigen Arten sowohl der diploiden wie der polyploiden Gruppe zurechnen, verändert sich der Gesamtpolyploidiegrad dadurch nicht sonderlich. Ich erhalte an Polyploiden für

\begin{tabular}{lccl} 
& \multicolumn{1}{c}{ Dikotyle } & Monokotyle & \multicolumn{1}{c}{ Angiospermen } \\
Centraleuropa & $47,7 \%$ (statt $47,3 \%)$ & $61,5 \%$ (statt $64,2 \%)$ & $50,7 \%$ (statt $50,9 \%$ ) \\
Schweden & $50,6 \%$ (statt $50,7 \%)$ & $69,8 \%$ (statt $72,7 \%$ ) & $56 \%$ (statt $56,9 \%$ ) \\
Groß-Britannien & $48,2 \%$ (statt $47,9 \%)$ & $65,8 \%$ (statt $68,5 \%)$ & $52,8 \%$ (statt $52,3 \%$ )
\end{tabular}

Bei den Zahlen von HASkELL (1952) würde ich den Polyploidiegrad erhalten für

$\begin{array}{ccc}\text { Dikotyle } & \text { Monokotyle } & \text { Angiospermen } \\ 46,3 \% & 65 \% & 50,6 \%\end{array}$

Das sind also die Zahlen, die auch HASKeLL selbst angibt.

Ich habe schon 1934 für Schleswig-Holstein die vorhandenen Angiospermen. daraufhin untersucht, ob sie ihre Hauptverbreitung nach Süden, Südost oder Südwest oder nach Norden besitzen. Ich hatte angegeben für die "Südpflanzen"

\begin{tabular}{|c|c|c|c|}
\hline e 82 & Mor & 22 & Angiospermen 104 \\
\hline $\begin{array}{l}\text { hrom. bek. } \quad \text { Polpl. } \\
56=63.3 \% \\
15=26,8 \%\end{array}$ & $\begin{array}{c}\text { chrom. bek. } \\
14=63,6 \%\end{array}$ & $\begin{array}{l}\text { Polpl. } \\
4=28,6 \%\end{array}$ & $\begin{array}{cc}\text { chrom. bek. } & \text { Polpl. } \\
70=67,3 \% & 19=27,1 \%\end{array}$ \\
\hline
\end{tabular}

Das Buch von Christiansen (1953) macht es mir möglich, jetzt genauer zu charakterisieren. Ich zähle dabei für die "Südpflanzen"

\begin{tabular}{|c|c|c|c|c|}
\hline \multicolumn{2}{|c|}{ Dikotyle 80} & \multicolumn{2}{|c|}{ Monokotyle 16} & Angiospermen 96 \\
\hline $\begin{array}{l}\text { chrom. bek. } \\
76=95 \%\end{array}$ & $\begin{array}{c}\text { Polpl. } \\
23=31,9 \%\end{array}$ & $\begin{array}{c}\text { chrom. bek. } \\
13=81,3 \%\end{array}$ & $\begin{array}{l}\text { Polpl. } \\
6=50 \%\end{array}$ & $\begin{array}{cc}\text { chrom. bek. } & \text { Polpl. } \\
89=92,7 \% & 29=34,5 \%\end{array}$ \\
\hline
\end{tabular}

Für die "Nordpflanzen" hatte ich angegeben

\begin{tabular}{cccccc}
\multicolumn{2}{c}{ Dikotyle 127} & \multicolumn{2}{c}{ Monokotyle 79} & \multicolumn{2}{c}{ Angiospermen 206} \\
chrom. bek. & Polpl. & chrom. bek. & Polpl. & chrom. bek. & Polpl. \\
$82=64,6 \%$ & $39=47,6 \%$ & $45=57 \%$ & $37=82,2 \%$ & $127=61,6 \%$ & $76=60 \%$
\end{tabular}

Jetzt werte ich nach CHRISTIANSEN

Dikotyle 127

chrom. bek. Polpl.

$126=99,1 \% \quad 70=64,8 \%$
Monokotyle 84

chrom. bek. Polpl.

$82=97,7 \% \quad 72=90 \%$
Angiospermen 211

chrom. bek. Polpl.

$208=98,6 \% \quad 142=75,5 \%$

Dabei habe ich hier für die "Südpflanzen" 5 Arten, für die "Nordpflanzen" 20 Arten mit diploiden und polyploiden Rassen nicht berücksichtigt.

Für die anderen von mir studierten Floren gebe ich im nachfolgenden 
die Zahl der Arten an, die Centraleuropa nicht mehr erreichen. Leider sind gerade diese noch sehr unvollständig auf ihre Chromosomenzahl hin untersucht. Das provisorische unserer Kenntnisse kommt dadurch besonders zum Ausdruck. Andererseits wird es aber vielleicht für weitere Studien stimulierend wirken. Ich erhalte danach

\section{Kykladen}

$\begin{array}{lccccc} & \% \text { der Ges. Fl. } & \text { chrom. bek. } & \text { Dipl. } & \text { Polpl. } & \text { D }+ \text { P } \\ \text { Dikotyle } & 695=74,1 & 263=37,8 \% & 177=67,3 \% & 74=28,1 \% & 12=4,6 \% \\ \text { Monokotyle } & 177=71,4 & 75=42,4 \% & 33=44 \% & 36=48 \% & 6=8 \% \\ \text { Angiospermen } & 872=73,5 & 338=38,7 \% & 210=62,1 \% & 110=32,5 \% & 18=5,4 \%\end{array}$

Unter Weglassung der Arten mit diploiden und polyploiden Rassen:

Dikotyle

Monokotyle

Angiospermen

\begin{tabular}{|c|c|c|}
\hline 251 & 177 & $5 \%$ \\
\hline & 33 & 36 \\
\hline $20=$ & $210=65,6 \%$ & $110=34,4$ \\
\hline
\end{tabular}

\section{Rumänien}

Dikotyle

Monokotyle

$\%$ der Ges. Fl. chrom. bek. $1019=37,8 \quad 319=31,3 \%$

$193=28,6 \quad 80=41,5 \%$

Angiospermen $1212=36,4$

\begin{tabular}{ccc} 
Dipl. & Polpl. \\
$209=65,7 \%$ & $106=33 \%$ \\
$39=48,75 \%$ & $35=43,75 \%$ \\
$298=62,2 \%$ & $141=35,3 \%$ \\
& \multicolumn{3}{c}{$\begin{aligned} \text { und polyploiden Ras } \\
\text { und }\end{aligned}$} \\
$209=66,4 \%$ & $106=33,6 \%$ \\
$39=52,7 \%$ & $35=47,3 \%$ \\
$248=63,8 \%$ & $141=36,2 \%$
\end{tabular}

$\mathrm{D}+\mathrm{P}$

$4=1,3 \%$

$6=7,5 \%$

$10=2,5 \%$

Unter Weglassung der Arten mit diploiden und polyploiden Rassen:

Dikotyle

Monokotyle

Angiospermen

$$
\begin{aligned}
& 315=30,9 \% \\
& 74=38,3 \% \\
& 389=32,1 \%
\end{aligned}
$$

\section{Ungarn}

$\begin{array}{lrrlrc}\text { Dikotyle } & 200=12,4 & 84=41,7 \% & 56=66,7 \% & 25=29,8 \% & 3=3,5 \% \\ \text { Monokotyle } & 35=8,2 & 19=54,3 \% & 12=63,2 \% & 7=36,8 \% & - \\ \text { Angiospermen } & 235=11,5 & 103=43,6 \% & 68=66 \% & 32=31,1 \% & 3=2,9 \%\end{array}$

Unter Weglassung der Arten mit diploiden und polyploiden Rassen:

Dikotyle

Monokotyle

Angiospermen

$$
\begin{aligned}
& 81=40,5 \% \\
& 19=54,3 \% \\
& 100=42,6 \%
\end{aligned}
$$

\section{Schweden}

Dikotyle Monokotyle Angiospermen

$$
\begin{aligned}
125 & =11,5 \\
71 & =16,2 \\
196 & =12,8
\end{aligned}
$$$$
97=77,6 \%
$$$$
56=78,9 \%
$$$$
153=78,1 \%
$$

$$
\begin{aligned}
60 & =61,9 \% \\
47 & =83,9 \% \\
107 & =70 \%
\end{aligned}
$$

\section{GroB-Britannien}

Dikotyle Monokotyle $280=21,1$ Angiospermen 
Nach Weglassung der Arten mit diploiden und polyploiden Rassen:

$\begin{array}{lclrl}\text { Dikotyle } & 148=52,9 \% & 77=52 \% & 71=48 \% & - \\ \text { Monokotyle } & 53=66,25 \% & 13=24,5 \% & 40=75,5 \% & - \\ \text { Angiospermen } & 201=55,8 \% & & 90=44,8 \% & 111=55,2 \%\end{array}$

Der Unterschied im Polyploidiegrade zwischen den nicht bis Centraleuropa gelangten Arten Schwedens und Groß-Britanniens ist sehr interessant. Der erheblich niedrigere Satz in letzterem Areal ist offensichtlich durch das atlantische Klima bedingt. Ein relativ großer Teil von mediterranen Arten hat sich in Groß-Britannien eingebürgert, der in Schweden fehlt. Für den Norden haben wir demgegenüber wieder mit einem Anstieg der Polyploiden zu rechnen.

\section{Faröer}

$\begin{array}{lllclc} & \% \text { Ges. Fl. } & \text { chrom. bek. } & \text { Dipl. } & \text { Polpl } & \text { D +P } \\ \text { Dikotyle } & 25=13,1 \% & 20=80 \% & 10=50 \% & 10=50 \% & - \\ \text { Monokotyle } & 12=11,2 \% & 12=100 \% & - & 12=100 \% & - \\ \text { Angiospermen } & 37=12,4 \% & \text { 32=86,5\% } & 10=31,25 \% & 22=68,75 \% & -\end{array}$

Island

$\begin{array}{lrrrrr}\text { Dikotyle } & 68=19,7 \% & 68=100 \% & 24=35,3 \% & 44=64,7 \% & - \\ \text { Monokotyle } & 39=24,8 \% & 39=100 \% & 3=7,7 \% & 36=92,3 \% & - \\ \text { Angiospermen } & 107=21,3 \% & 107=100 \% & 27=25,2 \% & 80=74,8 \% & -\end{array}$

\section{S. W. Grönland}

$\begin{array}{lllrll}\text { Dikotyle } & 66=36,5 \% & 43=65,2 \% & 15=34,9 \% & 28=45,1 \% & - \\ \text { Monokotyle } & 29=25,1 \% & 26=90 \% & 2=7,7 \% & 24=92,3 \% & - \\ \text { Angiospermen } & 95=32,5 \% & 69=72,6 \% & 17=24,6 \% & 52=75,4 \% & -\end{array}$

\section{Spitzbergen}

$\begin{array}{lllccl}\text { Dikotyle } & 67=68,4 \% & 45=67,2 \% & 13=28,9 \% & 32=71,1 \% & - \\ \text { Monokotyle } & 31=66 \% & 24=77,4 \% & 1=4,2 \% & 23=95,8 \% & - \\ \text { Angiospermen } & 98=67,5 \% & 69=70,4 \% & 14=20,3 \% & 55=79,7 \% & -\end{array}$

Es ist von großem Interesse, daß selbst im hohen Norden noch eine Anzahl indigener Diploider sich angesiedelt hat. Unter den bisher chromosomal bekannten finden sich neben der einzigen Monokotylen Puccinellia Vahliana $(n=7)$ noch 13 Dikotyle: Ranunculus lapponicus $(n=8)$, Saxifraga tenuis $(n=10)$, Chrysosplenium tetrandrum $(n=12)$, Cardamine bellidifolia $(n=8)$, Cochlearia groenlandica $(n=7)$, Melandryum apetalum $(n=12)$, Stellaria humifusa $(n=13)$, Minuartia rubella $(n=13)$, Cassiope tetragona $(n=13)$, Vaccinium microphyllum $(n=13)$, Polemonium boreale $(n=9)$, Mertensia maritima $(n=12)$ und Pedicularis hirsuta $(n=8)$. Polyploide Arten sind gerade für die Polemoniaceen, Boraginaceen und Scrophulariaceen nicht bekannt geworden. 


\section{Résumé}

1) Aus den mir bis Juli 1954 vorliegenden Daten errechnete ich folgende Prozentsätze für die diploiden und polyploiden Angiospermen, nachdem ich diejenigen Species, die möglicherweise diploide und polyploide Rassen im Gebiet besitzen, vorsichtshalber als " ununtersucht" fortließ. Dagegen wurden die Arten, für die nur in anderen Arealen derartige Rassen anzunehmen waren, in dem betreffenden infrage stehenden Gebiet eindeutig gewertet.

\begin{tabular}{|c|c|c|c|c|}
\hline & $\mathrm{qkm}$ & Artenzahl & $\begin{array}{l}\text { auf } 1000 \mathrm{qkm} \\
\text { (in ganzen Zahlen) }\end{array}$ & $\begin{array}{l}\text { chrom, bek. } \\
\text { ohne } D+P\end{array}$ \\
\hline Kykladen & 2695 & 1186 & 446 & $579=48,8 \%$ \\
\hline Rumänien & 237384 & 3365 & 15 & $2099=65,3 \%$ \\
\hline Ungarn & 93011 & 2039 & 22 & $1468=72 \%$ \\
\hline Centraleuropa & 695881 & 2909 & 4 & $2114=72,7 \%$ \\
\hline Schleswig-Holstein & 15658 & 1081 & 69 & $963=89,1 \%$ \\
\hline Schweden & 449199 & 1526 & 3 & $1322=86,6 \%$ \\
\hline Norrland & 261291 & 956 & 3 & $844=88,2 \%$ \\
\hline Groß-Britannien & 314281 & 1778 & 6 & $1436=80,7 \%$ \\
\hline Faröer & 1399 & 298 & 214 & $289=97 \%$ \\
\hline Island total & 102846 & 502 & 5 & $502=100 \%$ \\
\hline$"$ indigen & $"$ & 385 & - & $385=100 \%$ \\
\hline S. W. Grönland total & $\cdots$ & 292 & - & $251=86 \%$ \\
\hline$" \quad$ indigen & " & 262 & - & $223=85,1 \%$ \\
\hline Spitzbergen & 62105 & 145 & 2 & $122=84,1 \%$ \\
\hline
\end{tabular}

Es ist klar, daß in kleineren Arealen die Artenzahl pro $1000 \mathrm{qkm}$ entsprechend höher ist, als in größeren, die noch dazu keine all zu großen klimatischen Unterschiede aufweisen. In der Größe vergleichbare Areale wie Rumänien und Norrland (15:3) oder Ungarn und Island (22:5) zeigen besonders deutlich die Abnahme der Artenzahl von Süden nach Norden.

\begin{tabular}{lcccccc} 
& \multicolumn{6}{c}{ Diploid- und Polyploidgrad in Prozenten } \\
& \multicolumn{2}{c}{ Dikotyle } & \multicolumn{2}{c}{ Monokotyle } & \multicolumn{2}{c}{ Angiospermen } \\
& $\mathrm{D}$ & $\mathrm{P}$ & $\mathrm{D}$ & $\mathrm{P}$ & $\mathrm{D}$ & $\mathrm{P}$ \\
Kykladen & 67,6 & 32,4 & 46,8 & 53,2 & 63 & 37 \\
Rumänien & 57,6 & 42,4 & 37,6 & 62,4 & 53,2 & 46,8 \\
Ungarn & 55,4 & 44,6 & 37,6 & 62,4 & 51,4 & 48,6 \\
Centraleuropa & 52,7 & 47,3 & 35,8 & 64,2 & 49,1 & 50,9 \\
Schleswig-Holstein & 51,9 & 48,1 & 27,6 & 72,4 & 45,5 & 54,5 \\
Schweden & 50,7 & 49,3 & 27,3 & 72,7 & 43,1 & 56,9 \\
Norrland & 48,9 & 51,1 & 22 & 78 & 40,8 & 59,2 \\
Groß-Britannien & 52,1 & 47,9 & 31,5 & 68,5 & 46,7 & 53,3 \\
Faröer & 40,7 & 59,3 & 16,5 & 83,5 & 31,7 & 68,3 \\
Island total & 43,2 & 56,8 & 14 & 86 & 34,1 & 65,9 \\
$\quad$ " indigen & 36,9 & 63,1 & 12,8 & 87,2 & 28,1 & 71,9 \\
S. W. Grönland total & 39,1 & 60,9 & 12,5 & 87,5 & 29 & 71 \\
$\quad$ " indigen & 36,4 & 63,6 & 11 & 89 & 26 & 74 \\
Spitzbergen & 35 & 65 & 2,4 & 97,6 & 23,8 & 76,2
\end{tabular}


2) Für Schleswig-Holstein berechnete ich den Polyploidiegrad der Arten, die ihre Hauptverbreitung nach Süden, S. W. oder S.O. einerseits, nach Norden andererseits haben.

\begin{tabular}{lcccccc} 
& \multicolumn{2}{c}{ Dikotyle } & \multicolumn{2}{c}{ Monokotyle } & \multicolumn{2}{c}{ Angiospermen } \\
"Südpflanzen" & $\mathrm{D}$ & $\mathrm{P}$ & $\mathrm{D}$ & $\mathrm{P}$ & $\mathrm{D}$ & $\mathrm{P}$ \\
"Nordpflanzen" & 68,1 & 31,9 & 50 & 50 & 65,5 & 34,5 \\
& 35,2 & 64,8 & 10 & 90 & 24,5 & 75,5
\end{tabular}

3) Rechne ich für Centraleuropa, Schweden und Groß-Britannien die Arten mit diploiden und polyploiden Rassen in meiner Gruppierung mit, da hier am ersten eine Wahrscheinlichkeit besteht, daß tatsächlich beide Rassen auch im Gebiet enthalten sind, ergibt das

\begin{tabular}{lccccccc} 
& Zahl der untersuchten & \multicolumn{2}{c}{ Dikotyle } & \multicolumn{2}{c}{ Monokotyle } & \multicolumn{2}{c}{ Angiospermen } \\
& Einheiten & D & P & D & P & D & P \\
Centraleuropa & 2534 & 52,3 & 47,7 & 38,5 & 61,5 & 49,3 & 50,7 \\
Schweden & 1530 & 49,4 & 50,6 & 30,2 & 69,8 & 44 & 56 \\
Groß-Britannien & 1662 & 51,8 & 48,2 & 34,2 & 65,8 & 47,2 & 52,8
\end{tabular}

Für Island, S. W. Grönland und Spitzbergen sind keine Arten mit chromosomal verschiedenen Rassen aufgeführt, resp. sie sind von vorneherein als getrennte Arten aufgefaßt.

4) Eine Untersuchung derjenigen Arten, die nicht bis Centraleuropa gekommen sind, ergibt (wieder unter Weglassung derjenigen, die möglicherweise diploide und polyploide Rassen besitzen) für

\begin{tabular}{lccccccc} 
& Zahl der & \multicolumn{2}{c}{ Dikotyle } & \multicolumn{2}{c}{ Monokotyle } & \multicolumn{2}{c}{ Angiospermen } \\
& chrom. bek. & D & P & D & P & D & P \\
Kykladen & $338=38,7 \%$ & 70,5 & 29,5 & 47,8 & 52,2 & 65,6 & 34,4 \\
Rumänien & $389=32,2 \%$ & 66,4 & 33,6 & 52,7 & 47,3 & 63,8 & 36,2 \\
Ungarn & $100=42,9 \%$ & 69,1 & 30,9 & 61,3 & 38,7 & 68 & 32 \\
Schweden & $153=78,1 \%$ & 38,1 & 61,9 & 16,1 & 83,9 & 30 & 70 \\
Groß-Britannien & $360=57,5 \%$ & 52 & 48 & 24,5 & 75,5 & 44,8 & 55,2 \\
Faröer & $32=86,5 \%$ & 50 & 50 & - & 100 & 31,25 & 68,75 \\
Island & $107=100 \%$ & 35,3 & 64,7 & 7,7 & 92,3 & 25,2 & 74,8 \\
S. W. Grönland & $69=65,7 \%$ & 34,9 & 65,1 & 7,7 & 92,3 & 24,6 & 75,4 \\
Spitzbergen & $69=70,4 \%$ & 28,9 & 71,1 & 4,2 & 95,8 & 20,3 & 79,7
\end{tabular}

\section{Citierte Literatur}

Babcock, E. B. 1947. The genus Crepis. Univ. of Californ. Public. Bot. 21: $1 \mathrm{ff}$.

Christiansen, W. 1930. Ber. der D. botan. Gesellsch. 48: $276 \mathrm{ff}$.

- 1953. Neue kritische Flora von Schleswig-Holstein. Verlag A. Möller u. Söhne. Rendsburg. 532 pp.

Clapham, A. R., Tutin, T. G. u. Warburg, E. F. 1952. Flora of the British Isles. Univers. press. Cambridge. $1951 \mathrm{pp}$.

Engler, A.--Diels, L. 1936. Syllabus der Pflanzenfamilien. 11. Aufl. Verl. Gebr. Borntraeger. Berlin. 419 pp.

Favarger, A. 1946. Bullet. Soc. botan. Suisse. 56: $364 \mathrm{ff}$.

Felföldy, A. 1948. Acta Agrobotan. Hungar. 1 (2): 28.

Flovik, A. 1938. Hereditas 24: 265 ff.

- 1940. Hereditas 26: $430 \mathrm{ff}$.

Gardé, A. 1951. Genet Iber. 3: $133 \mathrm{ff}$. 
Gates, R. R. u. Latter, J. 1927. Journ. R. Microsc. Soc. 209 ff. Goodspeed, T. H. 1947. Proceed Nat. Acad. of Sci. 33: $158 \mathrm{ff}$.

Hagerup, O. 1928. Dansk bot. Arkiv. pp. 6(6) 27 pp.

- 1932. Hereditas 16: 19. ff.

Harada, I. 1942. Japan. Journ. of Genet. 18: $92 \mathrm{ff}$.

Haskell, G. 1952. Journ. of Ecology 40: $265 \mathrm{ff}$.

Jensen, H. W. 1951. Cellule 54: $133 \mathrm{ff}$.

Krause, O. 1931. Planta 13: $29 \mathrm{ff}$.

Löve, A. u. D. 1948. Chromosome Numbers of Northern Plants. Reykjavlk 133 pp.

- 1949. Portugal. Acta biol. Ser. A. (Festschr. R. R. Goldschmidt): 1273 ff.

de Mesquita Rodrigues, J. E. 1953. Diss. Coimbra 184 pp.

Müntzing, A. 1933. Hereditas 17: $233 \mathrm{ff}$.

Ownbey, G. B. 1951. Bull. Torrey Bot. Club 78: $233 \mathrm{ff}$.

Polunin, N. 1952. Journ. Linn. Soc. Botan. London 52: $349 \mathrm{ff}$.

Pólya, N. 1948. Archiv Biol. Hungar. Ser. II. 18: $349 \mathrm{ff}$.

- 1949. Acta Geobotan. Hungar. 6: 123 ff.

Rechinger, K. 1944. Denkschr. Akad. Wiss. Wien. Math. Naturw. Kl. 105: 1 Halbbd.

v. Soó. R. 1917. Acta Geobotan. Hungar. Ser. II. 6: $104 \mathrm{ff}$.

- 1951. A Magyar Növényvillág kézikönyve 1: u. I Budapest. Akad. Verlag. $532+1120$ pp.

Tarnavschi, J. T. 1948. Bull. Jardin et Musée Botan. Univ. Cluj, Roumania 28: Suppl. I, $130 \mathrm{pp}$.

Tischler, G. 1934. Englers Bot. Jahrb. 67: 1 ff.

- 1946. Zeitschr. f. Naturforsch. 1: 156 ff.

- 1950. Die Chromosomenzahlen der Gefäßpflanzen Mitteleuropas. Verlag W. Junk. Den Haag, 263 pp.

- 1954. Cytologia 19: 1 pp.

Turrill, D. D. 1936. Nature 137: $563 \mathrm{ff}$.

Weijer, J. 1952. Genetica 26: 1 ff.

v. Witsch, H. 1942. Österr. Bot. Zeitschr. 81: $108 \mathrm{ff}$.

Žukov, N. J. 1939. Vsesojužn Nauč. Issled. Inst. Tab. I. Makhar I Promysl A. J. Mikojana. Krasnodar, 198 ff. 